\title{
Detecção de Falhas em Processos Industriais Baseada no Algoritmo Evolutivo SOStream
}

\author{
Andressa Stéfany Silva de Oliveira* Rute Souza de Abreu* \\ Yuri Thomas Pinheiro Nunes* Luiz Affonso Guedes* \\ * Departamento de Engenharia de Computação e Automação, \\ Universidade Federal do Rio Grande do Norte, RN (e-mail: \\ astefanysoliveira@gmail.com).
}

\begin{abstract}
In this paper, we propose the use of the online learning clustering algorithm, named as SOStream, to fault detection of industrial process operation. In order to carry out this activity, it was necessary to adapt the original algorithm for avoiding fault data to be approximately normal data clusters. Aiming to evaluate the performance of the SOStream, we performed a case study using the industrial plant simulator Tennessee Eastman Process. The analysis was done taking that into account multivariable monitoring scenarios, obtaining results above $90 \%$ in accuracy and recall. This, it was observed that the analyzed approach obtained good performance to fault detection. In addition, the algorithm SOStream has characteristics very interesting for real-time fault detection, such as unsupervised and online learning, data-driven approach and recursive, which does not require storing the previous samples.
\end{abstract}

\section{Resumo:}

Neste artigo, propomos a utilização do algoritmo evolutivo de clusterização online SOStream para detecção de falhas em processos industriais. Para isso, a metodologia proposta inclui uma adaptação no algoritmo original para evitar que dados de falha sejam sejam aproximados para clusters de dados normais. A fim de validar a metodologia, um estudo de caso utilizando o simulador de plantas industriais Tennessee Eastman Process foi realizado. A análise foi feita em uma sequência contínua de dados, denominada de fluxo ou stream de dados, e de forma multivariável, obtendo-se resultados acima de $90 \%$ em precisão e recall. Essa abordagem evolutiva por ser baseada em dados e não supervisionada, torna o processo de detecção de falhas mais simples e com menor custo computacional quando comparado com as técnicas baseadas em modelos, além de possuir um dinamismo recursivo, não necessitando armazenar o histórico das amostras anteriores.

Keywords: Evolving Algorithms; Data Stream; Fault Detection; Clustering; Machine Learning. Palavras-chaves: Algoritmo Evolutivo; Fluxo de Dados; Detecção de Falhas; Clusterização; Aprendizagem de Máquina.

\section{INTRODUÇÃO}

Para atender às exigências como a alta produção com maior qualidade e também atender aos regulamentos de segurança e ambientais, os processos industriais modernos possuem inúmeros sensores com o intuito de monitorar e controlar continuamente as atividades de operação, resultando em um grande número de variáveis monitoradas.

Ao longo da operação do processo industrial há perturbações em que os controladores não conseguem manipular adequadamente, ocorrendo assim mudanças no comportamento do processo industrial. Muitas dessas pertubações são decorrentes de falhas em componentes do processo industrial (Chiang et al., 2001).

Em termos de segurança operacional, é importante que falhas em componentes do processo sejam identificadas em tempo hábil de modo a não levarem a planta a operar em regiões inadequadas, o que pode ocasionar perdas de produtividade, equipamentos ou até mesmo acidentes com trabalhadores e danos ao meio ambiente (Germano, 2017).

Visando melhorar os níveis de segurança operacionais de processos industriais, estudos com técnicas e métodos para detecção de falhas em operação vêm sendo propostos na literatura. Em geral, o desempenho dessas técnicas é medido por sua eficácia e eficiência em identificar a ocorrência de mudanças no processo. Na área de detecção de falhas, existem técnicas baseadas em modelo e técnicas baseadas em dados, onde esta última tem sido o alvo das pesquisas atuais (Yin et al., 2014). Técnicas baseadas em dados podem ser aplicadas com sucesso sem a necessidade de grande conhecimento matemático e físico do processo, diferentemente das baseadas em modelos que necessitam de modelos de referências adequados aos processos complexos e sua respectiva sintonia de parâmetros. Assim, as técnicas de detecção de falhas baseados em dados tendem ser mais factíveis quando se fala na implementação em cenários reais. 
Ainda sobre os métodos orientados a dados, Yin et al. (2014) informa que o custo da computação online é reduzido significativamente, obtendo também eficiência para aplicações industriais de larga escala. Em Yin et al. (2012), são apresentados alguns métodos baseado em dados para detecção de falhas, sendo eles: análise de componentes principais (PCA), mínimos quadrados parciais (PLS), análise de componentes independentes (ICA), Análise Discriminante de Fisher (FDA) e a subespacial assistida (SAP).

Além dessas abordagens, a atividade de detecção de falhas é caracterizada como um problema de clusterização de dados. A clusterização de dados é especificada como uma técnica de aprendizado de máquina não supervisionada, onde os clusters são determinados a partir de alguma característica ou propriedade em comum (Faceli et al., 2011). No contexto de detecção de falhas, existem dois agrupamentos de dados: um cluster contendo os dados advindos de operação normal do processo e outro cluster contendo dados decorrente de cenários de anormalidade na operação do processo industrial.

Atualmente existem algoritmos de clusterização que possuem o aprendizado de forma totalmente offline, online ou parcialmente online e offline. Para o caso dos métodos com aprendizagem offline, é preciso que haja um conhecimento prévio dos dados do processo para posteriormente serem utilizados na clusterização. Porém, segundo Kokate et al. (2018), seguir essa filosofia no ambiente industrial se torna inviável para detecção de falhas, visto que a mineração do fluxo de dados deve atender restrições como a resposta em tempo real, memória limitada e passagem única dos dados.

Em contrapartida aos algoritmos de aprendizagem offline, os com aprendizagem online recebem a cada instante de tempo um dado novo para ser processado. Em razão disso, quando falamos de métodos de clusterização desse tipo, de acordo com Kokate et al. (2018), há a utilização de características como volume, densidade, forma ou ordem temporal dos dados como base da formação do agrupamento, por ser impraticável armazenar todos os dados.

Dessa forma, os dados são processados à medida em que chegam nos algoritmos e descartados em seguida. Essa sequência contínua de dados é denominada de stream ou fluxo de dados (Isaksson et al., 2012). Nesse contexto, os algoritmos denominados evolutivos se apresentam bastante promissores, pois possuem um mecanismo de aprendizagem online e contínuo, ao decorrer do tempo, de modo a se adequar à forma do fluxo de dados. Além disto, os algoritmos evolutivos funcionam de forma recursiva, necessitando apenas dos dados mais recentes e de medidas descritivas, como a média e variância da distribuição dos dados.

Como a detecção de falhas de forma rápida e em tempo real é muito importante para a integridade da segurança operacional de processos industriais, objetiva-se neste trabalho, a investigação da adoção de algoritmos de clusterização com aprendizagem online para essa finalidade. Mais especificamente, será utilizado o método de clusterização online SOStream, o qual é um algoritmo de aprendizado evolutivo que atua na clusterização de dados de forma multivariada. Para validar a metodologia proposta, o método será aplicado a um conjunto de dados gerados através de simulação do benchmark Tennessee Eastman Process (TEP).
O restante deste trabalho está organizado da seguinte forma. A Seção 2 apresenta o algoritmo evolutivo SOStream. A Seção 3 aborda a metodologia utilizada. A Seção 4 apresenta a configuração experimental, apresentando resumidamente o simulador TEP e as métricas de desempenho adotadas para avaliação dos resultados. A Seção 5 apresenta os resultados obtidos com respectivas discussões. E, por fim, a Seção 6 apresenta as principais conclusões e indicações de trabalhos futuros.

\section{ALGORITMO EVOLUTIVO SOSTREAM}

O SOStream, proposto em Isaksson et al. (2012), é um algoritmo online baseado em densidade auto-organizável para a classificação de clusters com fluxo de dados. O algoritmo, originalmente, possui 4 parâmetros como entrada: o vetor de dados de entrada, $v(t)$, o fator de escala $\alpha$, o número mínimo de vizinhos MinPts e o limiar de fusão $\gamma$.

O algoritmo do SOStream originalmente é dividido em 7 sub-algoritmos, que descrevem as operações de criação, determinação dos vizinhos próximos, atualização, busca por interseção, esquecimento e fusão dos clusters.

Contudo, neste trabalho, o algoritmo de esquecimento dos clusters não será utilizado, visto que no processo de detecção de falhas a quantidade de ocorrência de falhas é significativamente menor do que a de não-falhas. Dessa forma, caso o fator de esquecimento seja introduzido no modelo, o SOStream poderá inferir que os clusters de falhas são menos relevantes e, por consequência, removêlos.

O ciclo de análise do algoritmo se dá da seguinte maneira: inicialmente é recebido o vetor de entrada d-dimensional, $\mathrm{v}(\mathrm{t})$ no tempo $\mathrm{t}$, onde $t=(1,2,3, \cdots, n)$. No cenário inicial, enquanto a quantidade mínima de clusters, também definida por MinPts, não é atingida, novos clusters vão sendo formados com apenas um ponto: $v(t)$. Assim, é criado para cada instante de tempo t um conjunto $\mathrm{M}(\mathrm{t})$, onde cada micro cluster possui informações a respeito do número de pontos relacionados a ele, $n_{i}$, o raio $r_{i}$ e o centroide $C_{i}$. Dessa forma cada cluster é representado pela tupla $N_{i}=\left(n_{i}, r_{i}, C_{i}\right)$.

Seguidamente, para cada novo vetor de entrada, é calculado a distância entre o ponto e o centroide de todos os clusters identificados anteriormente. Neste artigo, será adotada a distância Euclidiana e é eleito como cluster vencedor o que possuir a menor distância.

Caso $v(t)$ esteja dentro dos limites do raio do cluster vencedor (win.Radius), o vetor é adicionado ao cluster e o centroide é recalculado utilizando uma abordagem de média aritmética tradicional. Não satisfazendo essa condição, o $v(t)$ é identificado como falha e descartado se for verificado que $v(t)$ possui distância euclidiana maior que $p *$ win.Radius, caso contrário, é criado um novo cluster com esse vetor de entrada.

Os clusters vizinhos do vencedor têm seus centroides modificados para ficarem mais próximos do centroide deste cluster. Esta técnica é inspirada na atualização de pesos para o neurônio vencedor em uma Rede de Kohonen.

No cálculo de aproximação dos centroides é utilizado o parâmetro $\alpha$, que é equivalente a um fator de aprendizagem. 
Neste caso, quanto maior o seu valor, mais o algoritmo tentará aproximar os vizinhos ao vencedor.

A equação (1) representa o cálculo utilizado na atualização do centroide dos vizinhos do vencedor, sendo $\beta$ descrito na equação (2).

$$
\begin{gathered}
C_{i}(t+1)=C_{i}(t)+\alpha \beta\left(C_{w i n}(t)-C_{i}(t)\right) \\
\beta=e^{-\frac{d\left(C_{i}, C_{w i n}\right)}{2 r_{w i n}^{2}}}
\end{gathered}
$$

Após esta atualização, é verificado se há sobreposição dos vizinhos com o vencedor. Quando esta existe e é maior que o limiar de fusão $\gamma$, a operação é realizada. Essa dinâmica se repete para todos os pontos do fluxo de dados.

A operação de fusão define o centroide e o raio do novo cluster, de acordo com as equações (4) e (3), respectivamente.

$$
\begin{gathered}
N_{y}=\left(w_{i} a_{i}+w_{j} b_{i}\right) /\left(w_{i}+w_{j}\right) \\
r_{y}=\max \left\{d\left(C_{y}, C_{i}\right)+r_{i}, d\left(C_{y}, C_{i}\right)+r_{j}\right\}
\end{gathered}
$$

Na equação (4) os símbolos $w_{i}$ e $w_{j}$ são o número de pontos nos clusters $N_{i}$ e $N_{j}$, respectivamente. Além disso, $a_{i}$ e $b_{i}$ são as coordenadas dos centroides de cada cluster. Já na equação (4), $C_{y}$ representa o centroide do novo cluster. Da mesma maneira $C_{i}$ e $C_{j}$ são os centroides dos respectivos clusters $N_{i}$ e $N_{j}$.

\section{METODOLOGIA}

Visando a realização da detecção de falhas em variáveis de processo industrial, neste trabalho é utilizado o algoritmo evolutivo SOStream para a clusterização de dados online em dois tipos de clusters: um cluster para situações normais de operação e outro para situações de anormalidade.

Como mostrado no diagrama presente na Figura 1, o processo se inicia com a escolha dos parâmetros do algoritmo. Em seguida, é selecionado o conjunto de variáveis de processo que serão monitoradas para caracterização de cenário de falhas ou operação normal.

Neste trabalho, foram escolhidas variáveis que apresentavam desvio de seu nível normal, quando o distúrbio foi aplicado, porém, em situações reais é razoável que essa seleção seja determinada por um especialista do processo.

A partir deste ponto, os dados são enviados de maneira sequencial e passam a ser analisados pelo algoritmo. No SOStream, cada entrada é definida como um conjunto de $n$ variáveis no instante de tempo $t$. Assim, a cada instante de tempo o algoritmo recebe um vetor n-dimensional $(v(t))$, composto pelos valores das variáveis.

Para que o algoritmo decida se essa amostra representa uma situação de falha ou normalidade, algumas verificações são realizadas, conforme descrito na Seção 2. Contudo, além das operações já realizadas pelo algoritmo, a metodologia proposta realiza duas modificações adicionais, detalhadas na próxima subseção. Ao fim desta etapa, o método

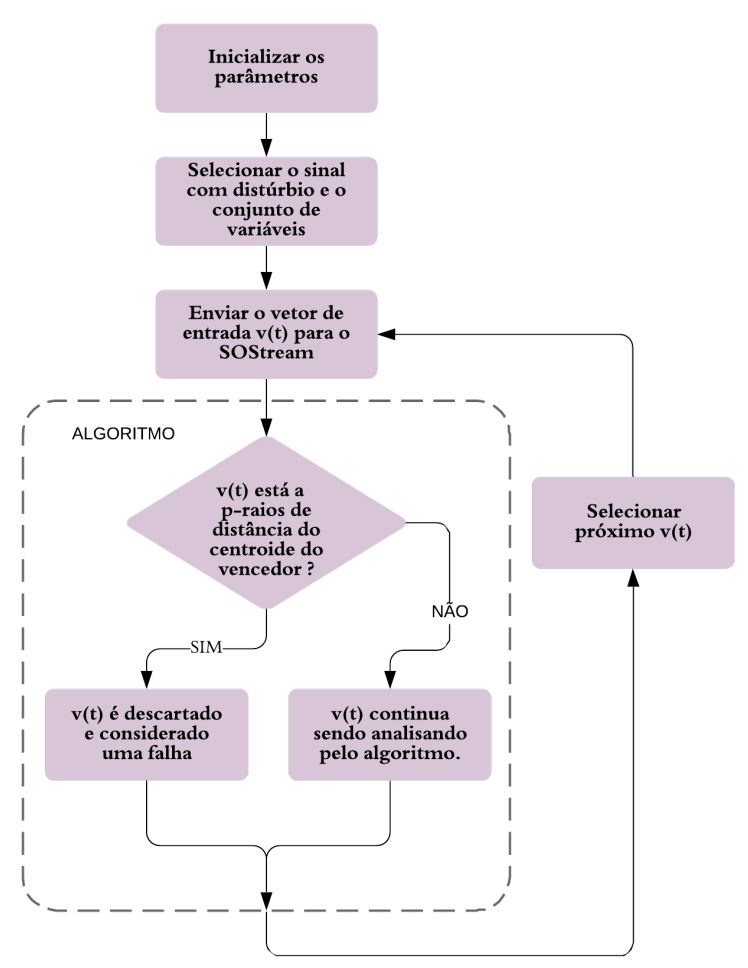

Figura 1. Diagrama de blocos.

aguarda a chegada da nova amostra para recomeçar o ciclo de processamento.

\subsection{Modificação do SOStream}

Para que o SOStream pudesse ser utilizado adequadamente para detecção de falhas, foi preciso realizar duas modificações no algoritmo original. A primeira consistiu na geração online de um vetor de detecção binário, onde o 0 indica normalidade e 1 indica a falha. Esse vetor é semelhante a um alarme industrial multivariável.

Além disso, uma nova verificação foi adicionada ao algorítimo: Se o $v(t)$ não estiver dentro do raio do cluster vencedor, há uma segunda verificação para saber se essa distancia entre eles é superior ao valor $p *$ win.radius, onde $p$ é um novo parâmetro de entrada do SOStream.

Em caso positivo, v(t) é descartado e é adicionado um 1 no vetor de detecção. Em caso negativo, um novo cluster é criado para $v(t)$ e o vetor de detecção recebe o valor 0 . Essa modificação pode ser vista no trecho de $6-9$, do Algoritmo 1.

\section{CONFIGURAÇÕES EXPERIMENTAIS}

Para a realização dos experimentos apresentados neste trabalho, um conjunto de dados gerados através do simulador Tennessee Eastman Process foi utilizado. A fim de simular um fluxo de dados, cada amostra do conjunto gerado foi introduzida individualmente no algoritmo SOStream adaptado. 


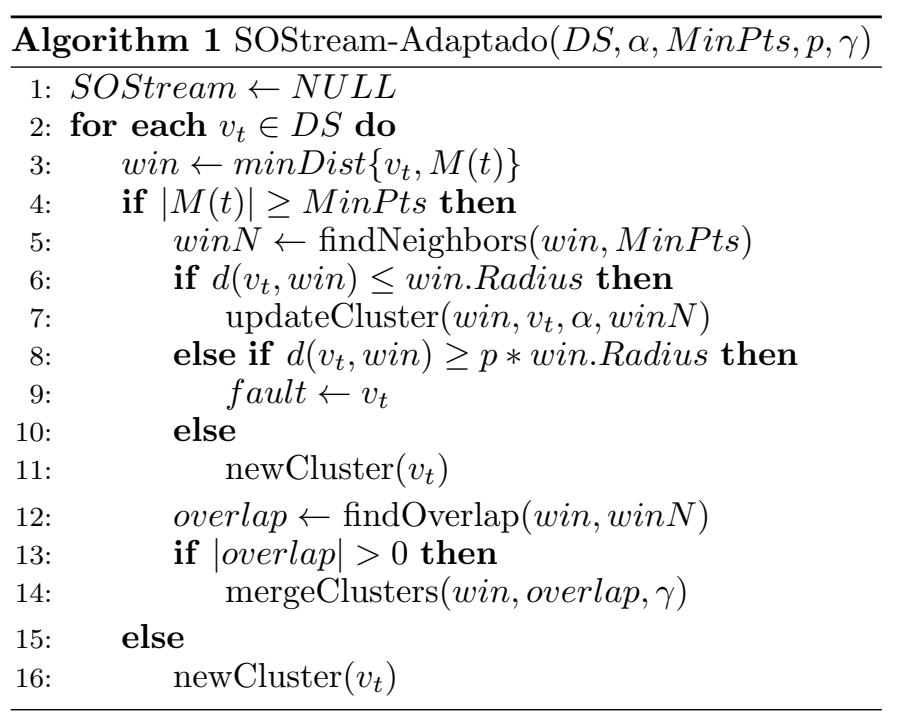

\subsection{Tennessee Eastman Process}

O Tennessee Eastman Process (TEP) é um simulador baseado em um processo químico industrial real, proposto em Downs and Vogel (1993). O processo consiste de cinco unidades principais, sendo elas o reator, o condensador de produto, um separador de vapor-líquido, um compressor de reciclagem e a decapagem. O processo é composto por 8 componentes, $A, B, C, D, E, F, G$ e $H$, com os quais a partir de quatro reações exotérmicas se formam dois produtos e dois subprodutos.

As equações de (5) a (8) definem essas reações.

$$
\begin{gathered}
A_{(g)}+C_{(g)}+D_{(g)} \rightarrow G_{(\text {liq })}, \text { Produto } 1 \\
A_{(g)}+C_{(g)}+E_{(g)} \rightarrow H_{(\text {liq })}, \text { Produto } 2 \\
A_{(g)}+E_{(g)} \rightarrow F_{(\text {liq) }}, \text { Subproduto } \\
3 D_{(g)} \rightarrow 2 F_{(\text {liq })}, \text { Subproduto }
\end{gathered}
$$

Sendo que as equações (5) e (6) descrevem a formação dos produtos a partir de quatro reagentes, e as equações (7) e (8) representam a formação dos subprodutos.

Além disso, o TEP possui 12 variáveis manipuladas e 41 variáveis de medição disponíveis para propósito de monitoramento ou controle. Dessas 41 variáveis de processo fornecidas para o monitoramento, 22 são variáveis de processo contínuas e 19 são variáveis de processo discretas. Para propósito de simulação de falhas e avaliação de métodos de controle e deteç̧ão de falha, o TEP possui ainda 20 distúrbios que podem ser aplicados durante as simulações (Downs and Vogel, 1993).

\subsection{Geração dos Dados}

Os dados gerados neste experimentos são produto de duas simulações de processo com 158 horas cada. A taxa de amostragem escolhida foi de 36 segundos. Em cada simulação foi aplicado um distúrbio, inciando na $101^{\mathrm{a}}$ hora com duração de 10 horas, seguido de 48 horas de estabilização. Na primeira simulação, foi aplicado o distúrbio IDV1. Este distúrbio provoca uma variação de passo na taxa de alimentação dos componentes A/C. Já na segunda simulação, o distúrbio escolhido foi o IDV13, que afeta a cinética das reações químicas.
As configurações de simulação e as variáveis selecionadas para análise neste experimento são mostradas na Tabela 1.

Tabela 1. Configurações de simulação.

\begin{tabular}{cc}
\hline Parâmetros & Configurações \\
\hline Duração (h) & 158 \\
Distúrbio & IDV1, IDV13 \\
Taxa de amostragem (s) & $36 \mathrm{~s}$ \\
Duração do distúrbio (h) & 10 \\
Tempo de Estabilização(h) & 48 \\
\hline
\end{tabular}

Tabela 2. Descrição das variáveis.

\begin{tabular}{lc}
\hline Variável & Descrição \\
\hline XMEAS01 & Alimentação do Componente A. \\
XMEAS10 & Taxa de eliminação de resíduos do compressor. \\
XMEAS21 & Temperatura de saída da água do resfriador. \\
\hline
\end{tabular}

\subsection{Métricas de Avaliação}

Para valiar a metodologia proposta, uma matriz de confusão foi gerada através dos resultados obtidos. A matriz de confusão expõe quatro possíveis saídas: verdadeiro positivo (VP), quando a entrada é uma falha e foi identificada como falha; falso negativo (FN), quando a entrada é uma falha e foi agrupada junto aos dados sem anormalidade, falso positivo (FP), quando a entrada é normal, mas foi identificada como falha e verdadeiro negativo (VN), quando a entrada é falha e foi agrupada junto aos dados com falha. (Max, 2016). A Tabela 3 mostra a estrutura desta matriz.

Tabela 3. Matriz de confusão.

\begin{tabular}{llcc}
\hline \multicolumn{3}{c}{ Predição } \\
\hline \multirow{3}{*}{ Real } & Falha & Não-Falha \\
& Não-falha & VP & FN \\
& FP & VN \\
\hline
\end{tabular}

Neste artigo, para o cálculo da matriz de confusão, as regiões de transições de normal para anormal e de anormal para normal foram ignoradas. Isso foi feito porque as variáveis de processo não reagem instantaneamente à mudança ocasionada pela ativação do distúrbio. Fazendo com que haja um atraso entre a ativação e a alteração do nível da variável. Da mesma forma, quando o distúrbio é desativado, a variável leva um tempo para perceber e se estabilizar.

No nosso caso, a região de transição da região normal para anormal foi definida como sendo o momento da ativação do distúrbio até a primeira identificação de falha realizada pelo algoritmo na região anormal do sinal. Já a transição da região anormal para normal foi definida do instante de desativação do distúrbio ao instante em que o algoritmo identifica, pela primeira, vez a região normal.

Dessa forma, as métricas aqui presentes, com exceção dos atrasos de ativação e estabilização, não contabilizam as regiões de transição. A partir da matriz de confusão as métricas: precisão, recall e F1 score, foram computadas, seguindo a definção mostrada nas equações a seguir. 


$$
\begin{gathered}
\text { precisao }=\frac{V P}{V P+F P} \\
\text { recall }=\frac{V P}{V P+F N} \\
F 1=\frac{2 * \text { precisao } * \text { recall }}{\text { precisao }+ \text { recall }}
\end{gathered}
$$

Além destas métricas, o tempo que o algoritmo leva pra detectar a falha no processo e o tempo que leva para perceber que não há mais falhas no processo são apresentados na seção de resultados.

\section{RESULTADOS E DISCUSSÃO}

Conforme descrito na metodologia, o algoritmo SOStream foi adaptado para realizar a detecção de falhas a partir do fluxo de dados. É importante ressaltar que os dados utilizados não sofreram nenhum tipo de pós-processamento, visto que em um processo real não é possível realizar esse tratamento, pois não temos o conhecimento de todo o conjunto de dados previamente.

Contudo, apenas para efeitos de visualização dos resultados, foi realizada uma normalização das variáveis antes da geração dos gráficos. Em todos os gráficos de resultados a curva binária na cor vermelha indicam o sinal de detecção obtido com aplicação do algoritmo. Já as curvas do sinal de processo são representadas na cor roxa. Além disso, há a inserção de uma faixa na cor cinza, indicando a região de aplicação do distúrbio.

Neste trabalho são analisados dois cenários de simulação. $\mathrm{Na}$ primeira simulação o distúrbio IDV1 é aplicado, enquanto na segunda o distúrbio IDV13 é utilizado. Para cada cenário de simulação, foram realizados 02 experimentos, onde se variou a parametrização do algoritmo SOStream, a fim de verificar sua robustez. Além disso, houve uma busca exaustiva para se chegar aos parâmetros utilizados.

\subsection{Cenário com Distúrbio IDV1}

Para o experimento 1 deste cenário, utilizou-se a configuração descrita na Tabela 4. Os resultados obtidos são mostrados na Figura 2. Note que na identificação feita pelo algoritmo, curva vermelha, há o reconhecimento da região em que ocorre falha, contudo existem alguns resultados falsos positivos neste gráfico, caracterizando que o algoritmo detectou falha na região de normalidade do processo.

Isso pode ter acontecido por ação do parâmetro $\gamma$, já que ele é o responsável pela realização da fusão dos clusters. Quando este parâmetro é muito pouco restritivo, o algoritmo tende a aglomerar dados mais distantes, podendo ocasionar resultados espúrios. Além disso, quando o número de vizinhos é muito pequeno, o método tende a manter o raio dos clusters pouco abrangente, já que o raio é definido como a MinPts-ésima menor distância entre o cluster vencedor e seus vizinhos.

De acordo com as métricas de avaliação expostas na Tabela 5 , temos que a precisão foi de 0,994 , o recall foi 1 e o $F 1-$ Score foi 0,997 . Apesar desses resultados
Tabela 4. Parametrizações dos experimentos IDV1.

\begin{tabular}{ccccc}
\hline Experimento & $\alpha$ & MinPts & $\gamma$ & $\mathbf{p}$ \\
\hline 1 & 0.3 & 3 & 0.02 & 1.5 \\
2 & 0.5 & 12 & 0.001 & 1 \\
\hline
\end{tabular}

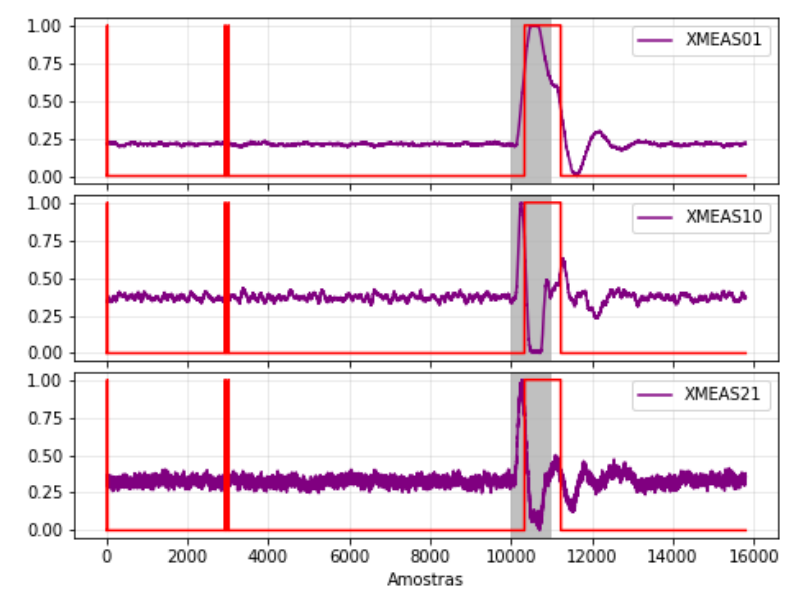

Figura 2. Experimento 1 com o distúrbio IDV1.

serem bons, houve uma demora de 330 amostras para o algoritmo detectar a falha, isto equivale a 3,30 horas. Além disso, o algoritmo demorou 226 amostras ou 2,26 horas para perceber a estabilização da planta, como descrito na Tabela 6 .

Visando melhorar o desempenho do algoritmo, no experimento 2, foram utilizados outros valores para os parâmetros do algoritmo SOStream, como pode ser visto na Tabela 4. No caso, foi diminuído o limiar de fusão, $\gamma$ e o valor de $p$, aumentado o fator o número de vizinhos, MinPts, e o fator de escala $\alpha$. Essa decisão se deve ao fato do valor de $p$ ter influência significativa na decisão do descarte de $v(t)$ como falha. Pois, quando o fator $p$ é 1 , qualquer $v(t)$ que não esteja dentro do raio do cluster vencedor será considerado uma amostra em falha e consequentemente será descartado. Ademais, quando o parâmetro $\alpha$ é aumentado, a aproximação dos vizinhos ao cluster vencedor é intensificada, facilitando as aglomerações de normalidade. Os resultados obtidos no experimento 2 podem ser visualizados na Figura 3.

O resultado das métricas de precisão, recall e F1-Score refente ao experimento 2 é descrito na Tabela 5. Com essa mudança de parâmetros, os resultados falsos positivos foram eliminados, além disso o atraso de tempo para a percepção da falha foi diminuído com relação ao experimento 1, passando de 330 amostras (3,3 horas) para 162 amostras (1,62 horas), uma variação percentual negativa de aproximadamente $50,9 \%$. Contudo, o tempo de detecção de ausência de falha aumentou de 226 amostras (2,26 horas) para 321 amostras (3,21 horas), portanto uma variação percentual positiva de aproximadamente $42 \%$.

Apesar desse aumento no tempo, podemos concluir que esse resultado é superior ao do experimento 1, pois em questões de segurança operacional, é mais importante que se detecte a presença de falhas do que a ausência destas. 




Figura 3. Experimento 2 com o distúrbio IDV1.

Os atrasos de tempo dos experimentos com o distúrbio IDV1 podem ser verificados na Tabela 6 .

\section{Tabela 5. Métricas do Distúrbio IDV1}

\begin{tabular}{cccc}
\hline Experimento & Precisão & Recall & F1-Score \\
\hline 1 & 0.994 & 1 & 0.997 \\
2 & 1 & 0.998 & 0.999 \\
\hline
\end{tabular}

Tabela 6. Atrasos de tempo do distúrbio IDV1, em amostras

\begin{tabular}{ccc}
\hline Experimento & Ativação & Desativação \\
\hline 1 & 330 & 226 \\
2 & 162 & 321 \\
\hline
\end{tabular}

\subsection{Cenário com Distúrbio IDV13}

Para o experimento 1 deste cenário, a parametrização do algoritmo SOStream é apresentada na Tabela 7. Os resultados obtidos quando a simulação utilizada no algoritmo teve a aplicação do distúrbio IDV13 são mostrados na Figura 4. Nela, se compararmos a região cinza, quando a falha está ativa, e a curva vermelha, que foi determinada pelo algoritmo, observa-se que é identificada a região em que ocorre a falha, como também, o algoritmo identifica erroneamente que ocorre falha na região de transição. O que acontece é que como a planta não se estabilizou ainda, o método continua identificando o comportamento anormal, que de fato está ocorrendo, apesar do distúrbio já ter sido desativado.

Na Tabela 9 as métricas de desempenho obtidas para esta parametrização são expostas. Novamente, o algoritmo mostrou bons resultados, porém mesmo com métricas altas, o método teve um tempo de atraso relevante, tendo sido 382 amostras ou 3,82 horas para perceber o distúrbio e 581 amostras ou 5,81 horas para perceber a estabilização do sinal.

O experimento 2 deste cenário utilizou os mesmos parâmetros usados no experimento 2 do cenário anterior para o algoritmo SOStream, com excessão do limiar de fusão $\gamma$, veja na Tabela 7. A Figura 5 mostra graficamente os resultados obtidos para o experimento 2 com o distúrbio

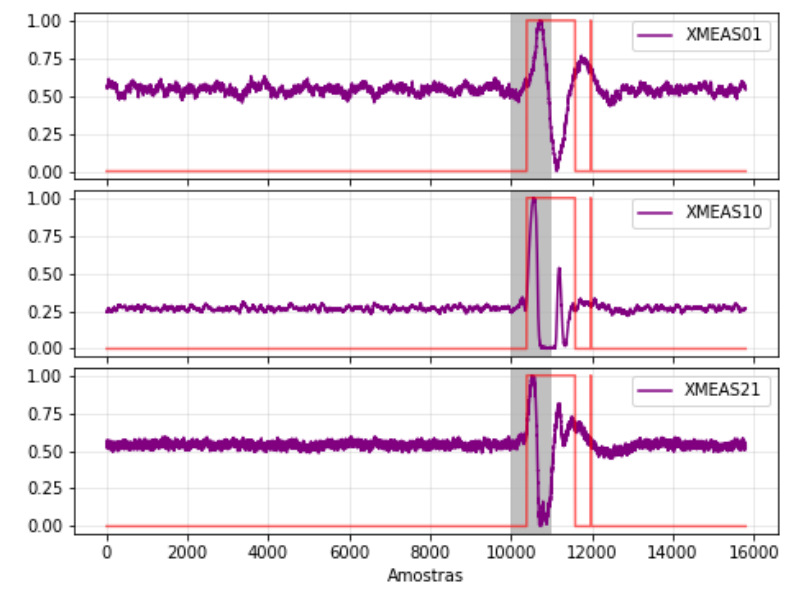

Figura 4. Experimento 1 com o distúrbio IDV13.

IDV13. A mudança nos parâmetros com relação ao experimento 1 fez com que a região classificada como alarme pelo algoritmo fosse menor, eliminando assim alguns falsos positivos. Contudo a detecção da falha foi significativamente mais lenta. Isso ocorreu porque o limiar de fusão, $\gamma$, foi aumentado fazendo com que a fusão se tornasse mais fácil, dessa forma o algoritmo acabou fundindo alguns clusters que já representavam estado de falha com cluster de dados sem falhas.

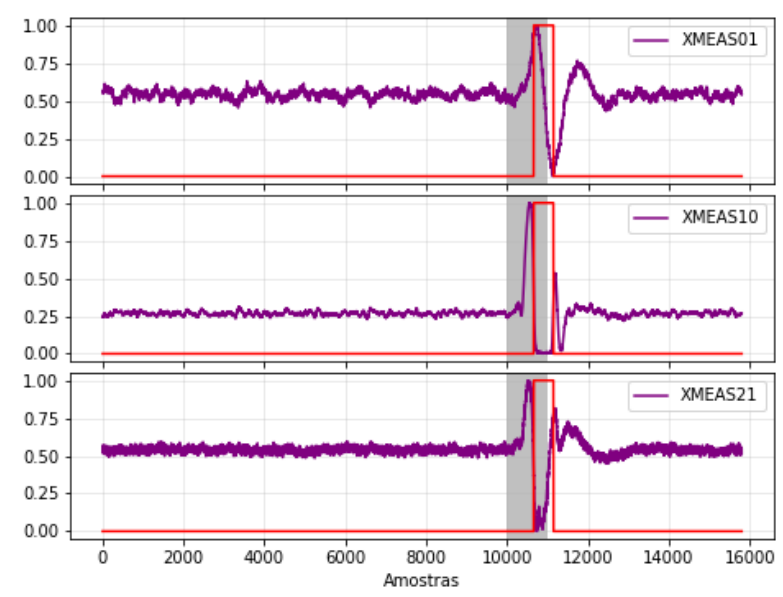

Figura 5. Experimento 2 com o distúrbio IDV13.

As métricas de desempenho obtidas nestes dois experimentos para detecção de falhas são apresentadas na Tabela 9. Apesar das métricas do experimento 2 serem maiores que a do experimento 1 , houve um aumento significativo do tempo de atraso.

A Tabela 8 mostra que o aumento percentual no tempo para percepção do distúrbio foi de aproximademte $71 \%$. Passando de 382 amostras (3,82 horas) para 656 amostras (6,56 horas). Já o atraso na percepção da estabilização, diminuiu passando de 581 amostras ( 5,81 horas) para 143 amostras (1,43 horas), uma variação percentual negativa de $75 \%$.

Dessa forma, podemos concluir que, para o distúrbio IDV13, a parametrização do algoritmo SOStream utilizada no experimento 2 apresentou melhor desempenho do que a parametrização utilizada no experimento 1 , já que a de- 
tecção deste foi mais rápida e o resultado de suas métricas não é significativamente inferior ao do experimento 1.

Tabela 7. Parametrizações dos experimentos IDV13.

\begin{tabular}{ccccc}
\hline Experimento & $\alpha$ & MinPts & $\gamma$ & $\mathbf{p}$ \\
\hline 1 & 0.35 & 5 & 0.0001 & 1.15 \\
2 & 0.5 & 12 & 0.01 & 1 \\
\hline
\end{tabular}

Tabela 8. Atrasos de tempo do distúrbio IDV13, em amostras

\begin{tabular}{ccc}
\hline Experimento & Ativação & Desativação \\
\hline 1 & 382 & 581 \\
2 & 656 & 143 \\
\hline
\end{tabular}

Tabela 9. Métricas do distúrbio IDV13.

\begin{tabular}{cccc}
\hline Experimento & Precisão & Recall & F1-Score \\
\hline 1 & 0.995 & 1 & 0.997 \\
2 & 1 & 1 & 1 \\
\hline
\end{tabular}

De modo geral, o SOStream obteve bom desempenho para identificação da região em distúrbio, o que consequentemente fez com que resultasse em boas métricas de avaliação. Porém, é importante ressaltar que na detecção de falhas o ganho nas métrica pode resultar em perda na agilidade, formando um contraponto que deve ser sempre analisado de acordo com o problema.

\section{CONCLUSÃO}

Neste artigo analisamos o desempenho do algoritmo evolutivo de clusterização online SOStream para a detecção de falhas em processos industriais. Desse modo, utilizamos de uma abordagem baseada em dados, tornando o processo de detecção mais simples e de menor custo computacional, com relação aos métodos baseados em modelos. Porém para realizar a detecção de falhas, o SOStream precisou passar por uma modificação, que consistiu na adição uma condição que verifica o quão distante o vetor de entrada está do cluster mais próximo a ele. Quando ele está mais distante que p-raios deste cluster, então o vetor de entrada é descartado e considerado como uma amostra em falha no processo.

Baseado nos resultados apresentados, podemos afirmar que o algoritmo SOStream apresentou bons resultados e várias características positivas para implementação em ambientes reais de operação de processos industriais, dada sua aprendizagem online e seu aspecto recursivo.

A aprendizagem online permite que se inicie o algoritmo apenas com dados de amostras provenientes de situações normais de operação, sem necessidade de apresentar ao algoritmo a priori nenhuma informação sobre dados de falhas. $\mathrm{O}$ aspecto recursivo permite o não armazenamento do histórico de amostras ou dados passados. Já que os clusters são representados apenas com o uso informações descritivas, como média, número de pontos e centroide.

Como trabalhos futuros, pretende-se fazer a análise de outras abordagens evolutivas no processo de detecção de falhas. Assim como, adicionar o processo de diagnostico de falhas também de maneira online.

\section{REFERÊNCIAS}

Chiang, L.H., Russel, E.L., and Braatz, R.D. (2001). Fault Detection and Diagnosis in Industrial Systems. Springer-Verlag London Berlin Heidelberg.

Downs, J.J. and Vogel, E.F. (1993). A plant-wide industrial process control problem. Computers and Chemical Engineering, 17(3), 245-255. doi:10.1016/0098-1354(93) 80018-I.

Faceli, K., Lorena, A.C., Gama, J., and Carvalho, A.C. (2011). Inteligência Artificial - Uma Abordagem de Aprendizado de Máquina. LTC, $1^{\mathrm{a}}$ edition.

Germano, A.L. (2017). Análise de Desempenho de Abordagens Orientadas a Fluxo de Dados Aplicadas à Detecção de Falhas de Processos Industriais. Dissertação de mestrado, Universidade Federal do Rio Grande do Norte, Natal - RN. URL https:// repositorio.ufrn.br/jspui/bitstream/123456789/ 24547/1/AmandaLucenaGermano $\left\{\backslash \_\right.$DISSERT . pdf.

Isaksson, C., Dunham, M.H., and Hahsler, M. (2012). SOStream: Self organizing density-based clustering over data stream. Lecture Notes in Computer Science (including subseries Lecture Notes in Artificial Intelligence and Lecture Notes in Bioinformatics), 7376 LNAI, 264278. doi:10.1007/978-3-642-31537-4_21.

Kokate, U., Deshpande, A., Mahalle, P., and Patil, P. (2018). Data Stream Clustering Techniques, Applications, and Models: Comparative Analysis and Discussion. Big Data and Cognitive Computing, 2(4), 32. doi:10. 3390/bdcc2040032.

Max, G. (2016). Seleção de Instâncias Baseado em Aprendizado de Métricas para $K$ Vizinhos Mais Próximos. Dissertação, Universidade Federal do Mato Grosso do Sul. URL http://repositorio. ufms.br:8080/jspui/bitstream/123456789/3000/1/ EduardoZ $\{\backslash$ ' $\{\mathrm{a}\}$ \}rateGuerreiroMax.pdf.

Yin, S., Ding, S.X., Haghani, A., Hao, H., and Zhang, P. (2012). A comparison study of basic data-driven fault diagnosis and process monitoring methods on the benchmark Tennessee Eastman process. Journal of Process Control, 22(9), 1567-1581. doi:10.1016/j.jprocont. 2012.06.009. URL http://dx.doi.org/10.1016/j. jprocont.2012.06.009.

Yin, S., Ding, S.X., Xie, X., and Luo, H. (2014). A Review on Basic Data-Driven Approaches for. IEEE Transactions on Industrial Electronics, 61(11), 64186428 . 